\title{
Inclusion of landslide tsunamis generation into a depth integrated wave model
}

\author{
C. Cecioni and G. Bellotti \\ University of Roma TRE, Civil Engineering Sciences Department (DSIC), Rome, Italy
}

Received: 4 June 2010 - Revised: 13 September 2010 - Accepted: 26 September 2010 - Published: 12 November 2010

\begin{abstract}
A numerical model based on the mild slope equation, suitable to reproduce the propagation of small amplitude tsunamis in the far field, is extended to reproduce the generation and the propagation of waves generated by landslides. The wave generation is modeled through a forcing term included in the field equation, which reproduces the effects of the movement of a submerged landslide on the fluid. The measurements of three dimensional laboratory experiments, which simulate tsunamis generated by landslide sliding along the flank of a conical island, are compared with the theoretical calculation results. The present approach is also compared with the similar method of Tinti et al. (2006) used for the generation of these waves in depth integrated model, and the different behavior when using frequency-dispersive and non-dispersive equations is highlighted.
\end{abstract}

\section{Introduction}

Tsunamis are long water waves generated by geophysical events, like earthquakes, landslides, submarine volcanic eruptions, etc. (Wiegel, 1955). These events produce sudden movements of the sea/ocean floor or of the water free surface, generating waves. Many different approaches and simplifications can be used to reproduce numerically/mathematically tsunamis. The most accurate models are those resolving the full three-dimensional equations of the fluid dynamics, e.g. the Navier-Stokes equations. Example of such models are those developed by Liu et al. (2005) and by Grilli et al. (1999, 2002). These models are computationally expensive and allow the simulation of the considered phenomena over relatively small geographical areas, typically of the order of a few $\mathrm{km}^{2}$.

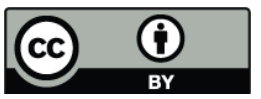

Correspondence to: C. Cecioni (ccecioni@uniroma3.it)
When studying the propagation of tsunamis over large areas, i.e. at the oceanic scales, depth integrated models are certainly more appropriate. They represent a good compromise between accuracy of the results and computational costs. Traditionally these models are based on the nonlinear shallow water equations, in view of the fact that tsunamis are considered to be very long waves if compared to the water depth where they propagate. However in the recent past it has become well accepted that this kind of waves is a wave packet, that in most cases may exhibit a frequency-dispersive behavior. The Boussinesq-type equations (BTE, Peregrine, 1967) have therefore become a standard tool for the simulation of tsunamis. These equations allow the reproduction of weakly nonlinear, weakly frequency-dispersive water waves, although recently new formulations of these equations can be applied to wider conditions. Moreover the wave frequencydispersive behavior becomes more evident for tsunamis generated by landslides, where the spatial extent of the source is usually not much larger than the water depth. One problem that arises when reproducing tsunamis with depth integrated models, is that, other than in shallow water approximation, it is not always possible to rigorously incorporate the effects of the movements of the sea floor into the equations, in order to reproduce the generation of waves due to earthquakes or landslides.

Jiang and LeBlonde (1994) have presented a numerical model to simulate the generation due to submarine mudslide, where the generated waves are governed by the nonlinear shallow water equations forced by the time variation of the sea bottom which is totally transferred to the free water surface. Tinti at al. (2001) have presented an analytical investigation of long water waves excited by rigid bodies sliding on the sea bottom, based on the linear shallow water equations. Their problem is based on the hyperbolic wave equation that includes a forcing term, defined as the second derivative in time of the varying water depth, modified by the passage of the landslide. Furthermore the paper of Tinti et al. (2006) 
shows the same technique applied in the framework of the nonlinear shallow water equations, and a filter function is introduced in the forcing term of the continuity equation in order to take into account when the slide length is no more much larger than the water depth. The continuity equation is formulated as follows

$\eta_{t}+\nabla_{\mathrm{h}} \cdot(\boldsymbol{v} h)=-h_{t} \frac{1}{\cos h\left(k_{\mathrm{s}} h\right)}$

where $\eta(\mathrm{x}, \mathrm{y}, t)$ is the free water surface, $v(\mathrm{x}, \mathrm{y}, \mathrm{z}, t)$ is the fluid particle velocity, $h(\mathrm{x}, \mathrm{y}, t)=h_{\mathrm{f}}(\mathrm{x}, \mathrm{y})-h_{l}(\mathrm{x}, \mathrm{y}, t)$ is the water depth function defined as the fixed sea floor depth $\left(h_{\mathrm{f}}\right)$ minus the thickness of the moving landslide $\left(h_{1}\right) . k_{\mathrm{s}}$ is the landslide length parameter, equal to $2 \pi / L_{\mathrm{S}}$, where $L_{\mathrm{S}}$ is the landslide length. Note that the water depth variation, due to the movements of the landslide, is filtered by means of a function which depends on the water depth and on the landslide length.

Alternatively, Watts et al. (2003) have proposed a tool namely the Tsunami Open and Progressive Initial Conditions System (TOPICS). Given the properties of the landslide, it allows calculation of proper initial conditions (in terms of water surface elevation and horizontal velocities of the fluid), that can be provided to depth-integrated models in order to represent the effects of the landslide on the waves. Moreover, Watts et al. (2005) presented numerical model application which simulates the generation and propagation of the Indian Ocean tsunami of 26 December 2004. They split the tsunami generating fault into four segments, and the vertical dislocation along each segment was given as input to the TOPICS software, which by means of the Okada formula (Okada, 1985) provide, as output, a characteristic wave length and tsunami initial amplitude. Then they simulate the tsunami propagation solving the higher-order Boussinesq equations in their FUNWAVE model. A further method is that developed by Lynett and Liu $(2002,2005)$, who have re-derived a Boussinesq-like set of equations that directly incorporates the effects of the movements of the bottom induced by the landslide. They have used scaling lengths that consider also the relative depth of the landslide, also providing guidance on the importance of frequency-dispersion and waves/landslide nonlinearity.

In this paper we describe and validate in more detail a method based on the depth integrated mild slope equation (hereinafter MSE), that we have already described in our previous paper Cecioni and Bellotti (2010). In that work we introduced heuristically a new function for the incorporation of the effects of the moving sea floor to reproduce the waves generation. The resulting forcing term which appears in the MSE is similar to that of Tinti et al. (2006), right hand side term of Eq. (1), but the filter function depends on the wave length instead of the landslide length. In Cecioni and Bellotti (2010) it was shown that the new technique is very accurate in the limits of mild slope sea bottom and small amplitude waves and landslide and it appears essential when ap- plying frequency-dispersive equations. The wave generation in the depth integrated model was validated with a full threedimensional numerical computations.

Here we make a step forward and we present the analytical derivation of the source term, starting from the depth integration of the Laplace equation with the appropriate moving bottom boundary conditions. Moreover we validate for the first time the model results using experimental data in a full three-dimensional layout. The data used are those presented by Di Risio et al. (2009) and Bellotti et al. (2009) and refer to the wave field generated around a conical island by a rigid body sliding down the flank of the island itself. The experimental layout was designed in order to reproduce approximately the case of Stromboli island (South Tyrrhenian Sea), scaled using the Froude law of 1:1000. The computations presented in this paper appear to be a very realistic study case. Stromboli is a volcanic island prone to tsunami generation along its own coast. On 30 December 2002 two tsunamis generated by landslides detached by the Sciara del Fuoco flank, attacked the coast devastating the small village of the island (Tinti et al., 2005).

In the next section the MSE with the forcing term is derived, while in the followings, first a short description of the physical model is given, then the comparison results are presented and discussed. Finally conclusions are given.

\section{Derivation of model equation}

The model equation can be obtained starting from the linear (small amplitude) water wave equations for an incompressible irrotational fluid on an uneven bottom

$$
\begin{array}{rlrl}
\nabla_{\mathrm{h}}^{2} \phi+\phi_{\mathrm{zz}}=0 & -h(\mathrm{x}, \mathrm{y}, t)<z & <0 \\
\phi_{\mathrm{z}}+\frac{1}{g} \phi_{\mathrm{tt}}=0 & z & =0 \\
\phi_{\mathrm{z}}+h_{t}+\nabla_{\mathrm{h}} \phi \cdot \nabla_{\mathrm{h}} h=0 & z & =-h(x, y, t),
\end{array}
$$

where $\phi(\mathrm{x}, \mathrm{y}, \mathrm{z}, t)$ is the velocity potential in the fluid, $h(\mathrm{x}, \mathrm{y}, t)$ is the water depth, defined as the fixed sea floor depth minus the landslide thickness, $h(\mathrm{x}, \mathrm{y}, t)=h_{\mathrm{f}}(\mathrm{x}, \mathrm{y})-$ $h_{1}(\mathrm{x}, \mathrm{y}, \mathrm{t}), g$ is the gravity acceleration, while $\nabla_{\mathrm{h}}$ is the differential operator which means the divergence in the horizontal coordinates $(\mathrm{x}, \mathrm{y})$ and the symbol $\cdot$ stays for the scalar product. All these variables are real and scalar. Equation (2) is the Laplace equation, Eq. (3) includes the dynamic and kinematic boundary conditions at the free water surface, while Eq. (4) is the bottom boundary condition which reproduces the sea floor movements allowing $h$ to varies in time. We follow the procedure described by Svendsen (2005), who starts from the Laplace equation and the free surface and bottom boundary conditions to derive the MSE, with the difference that here we take into account a moving sea floor. The solution of the given problem is assumed to be of the form

$\phi(\mathrm{x}, \mathrm{y}, \mathrm{z}, t)=\varphi(\mathrm{x}, \mathrm{y}, t) f(\mathrm{z})$

where $\varphi(\mathrm{x}, \mathrm{y}, t)$ is the velocity potential at the undisturbed 
free water surface $z=0$, which can be complex and it includes the effects of reflected waves; $f(z)$ is a function that describes how the kinematic field varies along the water depth and can be chosen as that resulting from the linear wave theory valid for harmonic waves propagating in constant depth, which however still holds locally for uneven bottom, i.e.

$f(z)=\frac{\cosh \left[k\left(h_{\mathrm{f}}+z\right)\right]}{\cosh \left(k h_{\mathrm{f}}\right)}$

where $k$ is the wave number, defined as $2 \pi / L$ with $L$ the wave length. In the cases of not constant depth, $h_{\mathrm{f}}$ and therefore $k$ vary with the horizontal coordinates, therefore $f=$ $f(\mathrm{x}, \mathrm{y}, \mathrm{z})$. However, if the mild slope assumption $\frac{\nabla_{\mathrm{h}} h_{\mathrm{f}}}{k h_{\mathrm{f}}} \ll 1$ is here introduced the variation of the function $f$ with the horizontal coordinates can be neglected if compared with the vertical one.

From the assumption (5) it comes that

$\phi_{\mathrm{zz}}=k^{2} \varphi \frac{\cosh \left[k\left(h_{\mathrm{f}}+z\right)\right]}{\cosh \left(k h_{\mathrm{f}}\right)}=k^{2} \phi$

therefore the Laplace equation (2) can be written as

$\nabla_{\mathrm{h}}^{2} \phi+k^{2} \phi=0$

The following considerations are made:

$$
\begin{array}{ll}
f(z)=1 & \text { at } z=0 \\
f_{\mathrm{z}}=k \tanh \left(k h_{\mathrm{f}}\right)=\frac{\omega^{2}}{g} & \text { at } z=0 ; \\
f_{\mathrm{z}}=0 & \text { at } z=-h ;
\end{array}
$$

In order to depth integrate the field equation (Laplace equation 2), here it is made use of the Gauss's Theorem, which states for one dimensional domain

$\int_{a}^{b} \frac{\partial \boldsymbol{v}}{\partial x} d x=\boldsymbol{v}(b)-\boldsymbol{v}(a)$

where $\boldsymbol{v}$ is a differentiable vector field. By considering a special vector field defined as $\boldsymbol{v}=\phi_{1} \nabla \phi_{2}$, where $\phi_{1}$ and $\phi_{2}$ are arbitrary differentiable scalar functions, the Gauss theorem can be written as

$$
\int_{a}^{b}\left[\phi_{1} \frac{\partial^{2} \phi_{2}}{\partial x^{2}}+\frac{\partial \phi_{1}}{\partial x} \frac{\partial \phi_{2}}{\partial x}\right] d x=\left[\phi_{1} \frac{\partial \phi_{2}}{\partial x}\right]_{b}-\left[\phi_{1} \frac{\partial \phi_{2}}{\partial x}\right]_{a}
$$

Equation 11 is known as Green's theorem. Interchanging $\phi_{1}$ and $\phi_{2}$ and subtracting the resulting equation from (11) gives

$\int_{a}^{b}\left[\phi_{1} \frac{\partial^{2} \phi_{2}}{\partial x^{2}}-\phi_{2} \frac{\partial^{2} \phi_{1}}{\partial x^{2}}\right] d x=\left[\phi_{1} \frac{\partial \phi_{2}}{\partial x}-\phi_{2} \frac{\partial \phi_{1}}{\partial x}\right]_{a}^{b}$

For the present purpose Eq. 12 is used with $x=z, \phi_{1}=$ $f(z)$ and $\phi_{2}=\phi(\mathrm{x}, \mathrm{y}, \mathrm{z}, t)$, therefore

$$
\begin{aligned}
& \int_{-h}^{0}\left(f \frac{\partial^{2} \phi}{\partial z^{2}}-\phi \frac{\partial^{2} f}{\partial z^{2}}\right) d z=\left[f \frac{\partial \phi}{\partial z}-\phi \frac{\partial f}{\partial z}\right]_{0} \\
& -\left[f \frac{\partial \phi}{\partial z}-\phi \frac{\partial f}{\partial z}\right]_{-h}
\end{aligned}
$$

Substituting the Laplace equation (2) in the first term at the left hand side (LHS), and the boundary conditions at $z=0$ and $z=-h$ (3 and 4) and using Eqs. (9) in the right hand side (RHS) terms, then, after changing the sign, Eq. (13) becomes

$\int_{-h}^{0}\left(f \nabla_{\mathrm{h}}^{2} \phi+k^{2} f \phi\right) d z=\frac{1}{g} \varphi_{\mathrm{tt}}+\varphi \frac{\omega^{2}}{g}-\left[f h_{t}\right]_{-h}$

$-\left[f \nabla_{\mathrm{h}} h \cdot \nabla_{\mathrm{h}} \phi\right]_{-h}$

the LHS can be seen as the integration over the depth of the field equation. Considering that

$\nabla_{\mathrm{h}} \phi=\nabla_{\mathrm{h}}(\varphi f)=f \nabla_{\mathrm{h}} \varphi+\varphi \nabla_{\mathrm{h}} f$

and

$\nabla_{\mathrm{h}}^{2} \phi=f \nabla_{\mathrm{h}}^{2} \varphi+2 \nabla_{\mathrm{h}} \varphi \cdot \nabla_{\mathrm{h}} f+\varphi \nabla_{\mathrm{h}}^{2} f$

using the expression (15) for the last term of the RHS and expression (16) for the first term of the LHS, Eq. (14) becomes

$\int_{-h}^{0}\left(f^{2} \nabla_{\mathrm{h}}^{2} \varphi+2 f \nabla_{\mathrm{h}} f \cdot \nabla_{\mathrm{h}} \varphi+f \varphi \nabla_{\mathrm{h}}^{2} f+k^{2} f^{2} \varphi\right) d z=$
$\frac{1}{g}\left(\varphi_{\mathrm{tt}}+\omega^{2} \varphi\right)-\frac{1}{\cosh \left(k h_{\mathrm{f}}\right)} h_{t}-\left[f \nabla_{\mathrm{h}} h \cdot\left(f \nabla_{\mathrm{h}} \varphi+\varphi \nabla_{\mathrm{h}} f\right)\right]_{-h}$

Now incorporating the first two terms of the LHS of Eq. (17) follows

$$
\begin{aligned}
& \int_{-h}^{0} \nabla_{\mathrm{h}} \cdot\left(f^{2} \nabla_{\mathrm{h}} \varphi\right) d z+\left[f^{2} \nabla_{\mathrm{h}} h \cdot \nabla_{\mathrm{h}} \varphi\right]_{-h}+\varphi k^{2} \int_{-h}^{0} f^{2} d z \\
& =-\int_{-h}^{0} \varphi f \nabla_{\mathrm{h}}^{2} f d z-\frac{1}{\cosh \left(k h_{\mathrm{f}}\right)} h_{t}-\varphi \nabla_{\mathrm{h}} h \cdot\left[f \nabla_{\mathrm{h}} f\right]_{-h} \\
& +\frac{1}{g}\left(\varphi_{\mathrm{tt}}+\omega^{2} \varphi\right)
\end{aligned}
$$

Applying the Leibniz's rule for the first two terms on the LHS and knowing that

$$
\int_{-h}^{0} f^{2} d z=\frac{c c_{\mathrm{g}}}{g}
$$

where $c$ and $c_{\mathrm{g}}$ are respectively the phase and the group velocities, by multiplying Eq. (18) for $g$ it results

$$
\begin{aligned}
& \nabla_{\mathrm{h}} \cdot\left(c c_{\mathrm{g}} \nabla_{\mathrm{h}} \varphi\right)+\varphi k^{2} c c_{\mathrm{g}}-\varphi_{\mathrm{tt}}-\omega^{2} \varphi+h_{t} \frac{g}{\cosh \left(k h_{\mathrm{f}}\right)} \\
& =-g \varphi\left\{\int_{-h}^{0} f \nabla_{\mathrm{h}}^{2} f d z+\nabla_{\mathrm{h}} h \cdot\left[f \nabla_{\mathrm{h}} f\right]_{-h}\right\}
\end{aligned}
$$

Rigorously the identity of Eq. (19) is obtained for a single frequency of the wave spectrum, consequently Eq. (20) is valid for monochromatic waves, or can be seen as representative of narrow banded spectra sea state around a carrying frequency. The RHS terms of Eq. (20) can be shown to be $O\left(\left(\nabla_{\mathrm{h}} h\right)^{2}, \nabla_{\mathrm{h}}^{2} h\right)$. Therefore, as we introduced the mild slope assumption above, i.e.: by allowing Eq. (6), $\nabla_{\mathrm{h}} h \ll k h$ and it can be argued that the RHS terms «LHS terms. Similarly, $\nabla_{\mathrm{h}}^{2} h \ll \nabla_{\mathrm{h}} h$, which is a natural additional assumption because $\nabla_{\mathrm{h}}^{2} h=O\left(\nabla_{\mathrm{h}} h\right)$ can only occur over short distances 
without changing $O\left(\nabla_{\mathrm{h}} h\right)$. This means that the RHS terms are $\ll$ of all the others terms, we therefore get

$\varphi_{\mathrm{tt}}-\nabla_{\mathrm{h}} \cdot\left(c c_{\mathrm{g}} \nabla_{\mathrm{h}} \varphi\right)+\left(\omega^{2}-k^{2} c c_{\mathrm{g}}\right) \varphi=-\frac{g}{\cosh \left(k h_{\mathrm{f}}\right)} h_{t}$

which is the hyperbolic version of the MSE in terms of fluid velocity potential and is usually referred to as the "timedependent mild-slope equation", allowing the simulation in the time-domain of the wave propagation. To obtain the MSE in terms of the free surface elevation $\eta$, Eq. (21) needs to be differentiated with respect to time.

$\varphi_{\mathrm{ttt}}-\nabla_{\mathrm{h}} \cdot\left(c c_{\mathrm{g}} \nabla_{\mathrm{h}} \varphi_{t}\right)+\left(\omega^{2}-k^{2} c c_{\mathrm{g}}\right) \varphi_{t}=-\frac{g}{\cosh \left(k h_{\mathrm{f}}\right)} h_{\mathrm{tt}}$

and then use the dynamic boundary condition at the free surface

$\eta=-\frac{1}{g} \varphi_{t}$

from which we obtain that $\varphi_{t}=-g \eta$ and $\varphi_{\mathrm{tt}}=-g \eta_{t}$, those expressions can be substituted into Eq. (22) to get, after dividing by $g$

$-\eta_{\mathrm{tt}}+\nabla_{\mathrm{h}} \cdot\left(c c_{\mathrm{g}} \nabla_{\mathrm{h}} \eta\right)-\left(\omega^{2}-k^{2} c c_{\mathrm{g}}\right) \eta=-\frac{1}{\cosh \left(k h_{\mathrm{f}}\right)} h_{\mathrm{tt}}$

which is the hyperbolic version of the MSE in terms of water free surface elevation. Note that if the phase and group velocities are evaluated in the shallow water limit as $c=c_{\mathrm{g}}=\sqrt{g h}$, then Eq. (24) reduces to the governing equation for forced long waves

$\eta_{\mathrm{tt}}-\nabla_{\mathrm{h}} \cdot\left(g h \nabla_{\mathrm{h}} \eta\right)=\frac{1}{\cosh \left(k h_{\mathrm{f}}\right)} h_{\mathrm{tt}}$

As said the time dependent MSE is not able to reproduce broad banded spectra sea state, due to the fact that Eq. (24) contains some coefficients which have to be calculated for each wave frequency component. By employing the spectral approach, the model can on the contrary cover a broad spectrum wave field, typical of tsunamis, since for each wave frequency a dedicated elliptical equation is solved and, due to the linearity of the equations, superimposition of all the solutions is allowed.

The elliptic version of the MSE can be obtained by taking the Fourier Transform of Eq. (24), then it comes

$\nabla_{\mathrm{h}} \cdot\left(c c_{\mathrm{g}} \nabla_{\mathrm{h}} N\right)+k^{2} c c_{\mathrm{g}} N=-\frac{1}{\cosh \left(k h_{\mathrm{f}}\right)} f f t\left(h_{\mathrm{tt}}\right)$

where $N(\mathrm{x}, \mathrm{y}, \omega)$ is the Fourier Transform of $\eta(\mathrm{x}, \mathrm{y}, t)$. Equation (26) is similar to the elliptic MSE as derived by Berkhoff (1972), but it includes a source term of generating waves. Once Eq. (26) is solved, one for each frequency $\omega$, with the appropriate boundary conditions, the result in the time domain can be achieved by taking the Inverse Fourier Transform of $N(\mathrm{x}, \mathrm{y}, \omega)$ to obtain $\eta(\mathrm{x}, \mathrm{y}, t)$. For details see Bellotti et al. (2008).

\section{Physical model description}

The results of physical model experiments, which aim at studying the tsunami wave field generated by landslide, have been used in order to validate the tsunami generation numerical model. The physical model was built in a large wave tank at the Research and Experimentation Laboratory for Coastal Defense (LIC) of the Technical University of Bari, Italy, in cooperation with the Environmental and Maritime Hydraulics Laboratory Umberto Messina (LIAM) of the University of L'Aquila, Italy.

The laboratory experiments (Di Risio et al., 2009) simulate a landslide body falling on the flank of a conical island, built in order to approximately reproduce in scale 1:1000 the Stromboli island, in South Tyrrhenian Sea, Italy (Tinti et al., 2005). The physical model consists in a wave tank, $30.00 \mathrm{~m}$ wide, $50.00 \mathrm{~m}$ long and $3.00 \mathrm{~m}$ deep; at the center of the tank is placed a conical island, built using PVC sheets (thickness $0.01 \mathrm{~m}$ ) and sustained by a steel frame, with a radius of $4.47 \mathrm{~m}$ at the tank bottom level. The slope of the flanks of the island is 1:3 (1 vertical, 3 horizontal). In Fig. 1 it can be seen a layout of the wave tank with the conical island (left figure) and a picture of the landslide model on the top of the island (right figure).

Experiments have been carried out varying the water depth, and consequently the shoreline curvature radius, and by varying the initial distance of the landslide from the undisturbed shoreline. The landslide model is a rigid body, with the shape of half of the ellipsoid described by the equation $x^{2} / a^{2}+y^{2} / b^{2}+z^{2} / c^{2}=1$, where $a=0.2 \mathrm{~m}, b=0.4 \mathrm{~m}$ and $c=0.05 \mathrm{~m}$, for a total volume $V=0.0084 \mathrm{~m}^{3}$. The landslide is constrained to slide down the inclined island surface by means of rails. Traditional resistive gauges were employed to register the instantaneous vertical displacement of the free surface. All the signals have been acquired simultaneously at a frequency of $1000 \mathrm{~Hz}$. The relative positions of all the gauges can be found in Fig. 2 and in Table 1. In Table 1 the gauges position is calculated in polar coordinates with the origin in the impact point (see Fig. 2) and the angular position taken counterclockwise from the landslide motion direction.

As an exercise we have superimposed the laboratory experiments layout scaled of 1000 times using the Froude law, to the map of Stromboli island (Fig. 3). The Figure gives an idea of the similarities between the two problems. The scaled position of the surface level measurements points is also reported. Given the similarity between laboratory and field cases, Bellotti et al. (2009) have investigated on the feasibility of Tsunami Early Warning Systems for small volcanic islands focusing on warning of waves generated by landslides at the coast of the island itself. 

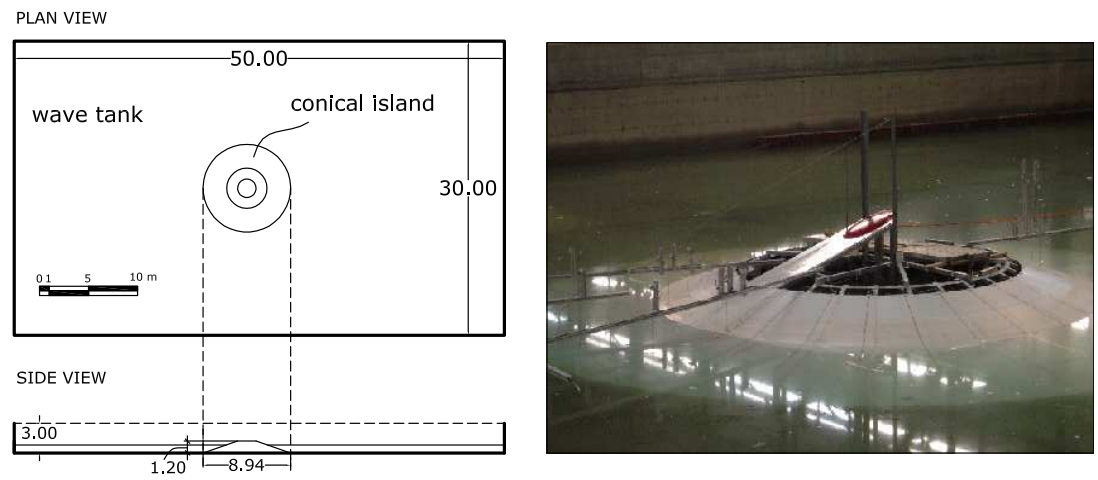

Fig. 1. Sketch of the layout of the laboratory experiments (left, measures in $\mathrm{m}$ ) and picture of the landslide and island models (right).

Table 1. Angular and radial position of Sea Level gauges: the point where the landslide impacts the water is taken as the origin, and the angular position is taken counterclockwise from the landslide motion direction (see Fig. 2).

\begin{tabular}{lcc}
\hline Gauge name & $\begin{array}{c}\text { Angular position } \\
\theta\left(^{\circ}\right)\end{array}$ & $\begin{array}{c}\text { Radialposition } \\
r(\mathrm{~m})\end{array}$ \\
\hline $12 \mathrm{~S}$ & 54.3 & 0.63 \\
$11 \mathrm{~S}$ & -84.4 & 0.76 \\
$20 \mathrm{~S}$ & 44.9 & 0.92 \\
$7 \mathrm{~S}$ & 29.2 & 1.82 \\
$15 \mathrm{~S}$ & -0.3 & 2.37 \\
$24 \mathrm{~S}$ & 0.8 & 4.55 \\
$22 \mathrm{~S}$ & 19.5 & 4.79 \\
$16 \mathrm{~S}$ & -29.7 & 4.91 \\
\hline
\end{tabular}

\section{Comparison of physical and numerical model results}

We have reproduced numerically the experiments described by Di Risio et al. (2009). Here we describe in details the results of just one test, defined by the off-shore constant water depth of $0.80 \mathrm{~m}$, and consequently the shoreline radius of $2.07 \mathrm{~m}$, and characterized by an aerial landslide which falls from a distance of $\zeta=0.30 \mathrm{~m}$ from the undisturbed shoreline. All the laboratory experiments tested reproduce aerial landslides, while the numerical model is able to simulate just underwater sea floor movements. Therefore the entering phase of the landslide, when it acts as a piston and transfers impulsive energy to the fluid, it is not reproduced numerically.

The computations have been carried out on a twodimensional domain, sketched in Fig. 4. In order to save computational costs, not all the wave tank was modeled. Taking advantage of the fact that the problem is symmetric across the landslide motion direction, we reproduced only one half of the domain, i.e. the one on the left side, following the landslide trajectory. Therefore the position of the gauges

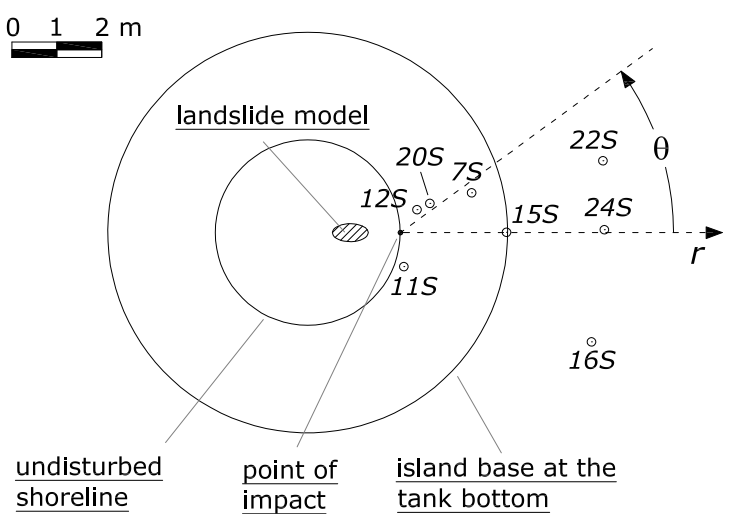

Fig. 2. Layout of the laboratory gauges positions.

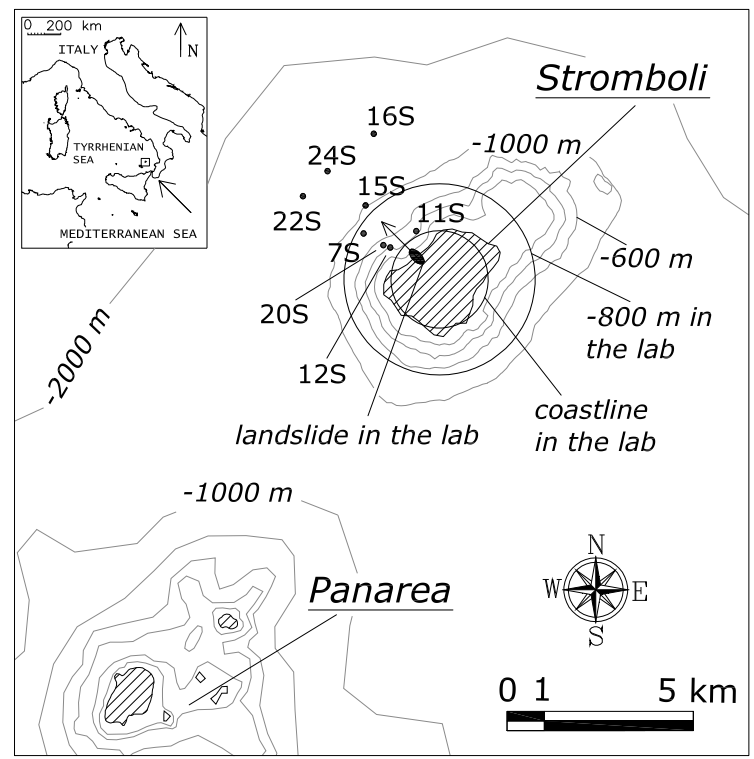

Fig. 3. Sketch of the bathymetry around Stromboli and Panarea islands (South Tyrrhenian Sea), where we have superimposed the layout of the physical model, with the gauges positions, scaled of 1000 times using the Froude law. 


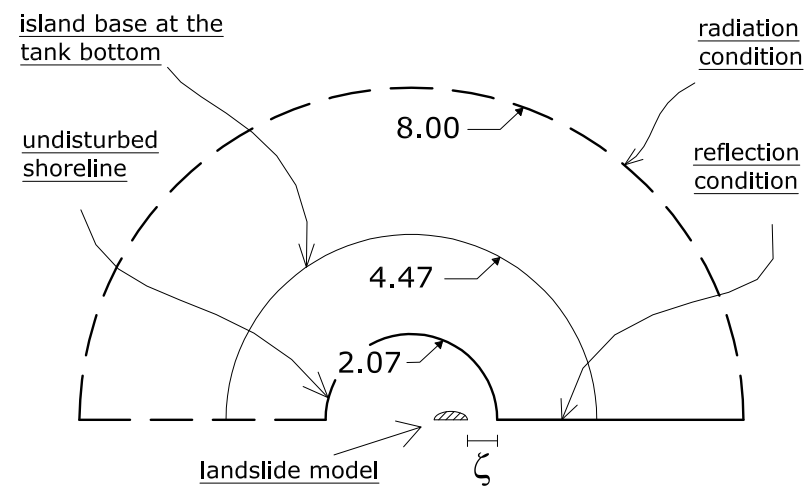

Fig. 4. Numerical domain of the depth integrated model. The numbers $2.07,4.47$ and 8.00 express the radii in meters of respectively the undisturbed shoreline, the island base at the tank bottom, and the external circular boundary.

located on the other side, i.e. $180^{\circ}<\theta<360^{\circ}$ (see Fig. 2 and Table 1), have been mirrored across the landslide motion direction. Furthermore, the numerical domain is limited by a circular border located at a distance of $8.00 \mathrm{~m}$ from the island centre.

At the internal circular border (the undisturbed shoreline) the reflection condition is imposed and a minimum water depth of $0.0001 \mathrm{~m}$ is guaranteed. Along the external circular boundary the waves are allowed to freely exit the computational domain. The landslide falls down in the direction of the right-bottom border, as sketched in Fig. 4. At that boundary the full reflection condition is imposed in order to take into account the symmetric half of the domain which is not simulated. For more details about the implementation of the boundary conditions, the reader is addressed to the paper of Bellotti et al. (2008). The water depth function $h(\mathrm{x}, \mathrm{y}, t)$, which takes into account the sea floor motion, due to the landslide, is calculated by knowing the landslide shape and movement. The second derivative in time of the function, $h_{\mathrm{tt}}(\mathrm{x}, \mathrm{y}, t)$, is carried out using a numerical approximation and its Fourier transform is applied, in order to insert it into the field Eq. (26).

The time series reproduced is of $50 \mathrm{~s}$, using a $\Delta t$ of $0.01 \mathrm{~s}$, resulting in a total of 5000 time steps, that correspond to the same number of frequency components. In order to save computational costs just the first 100 angular frequencies are solved, ranging between $2 \pi \cdot 0.02<\omega<2 \pi \cdot 2$. The higher frequency corresponds, for the considered water depth, to a wave length which slightly varies around the value of $0.4 \mathrm{~m}$. Therefore the numerical simulation, carried out with a finite element method, uses triangular linear elements with maximum size of $0.04 \mathrm{~m}$, in order to ensure the minimum of 10 points per wave length.
Figure 5 shows the comparison of the free water surface elevation at the 8 sea-level gauges (see Fig. 2) as measured in the physical model (thick black lines) and as calculated by the numerical model (thin black lines). In the same figure are also presented the results of other numerical simulations (thin red lines) which refer to the solution of the elliptic MSE but using the source term as the one proposed by Tinti et al. (2006). In this case the right hand side of Eq. (26) contains $k_{\mathrm{s}}$ instead of $k$. The panels are ordered with the distance from the impact point, i.e. where the landslide firstly impacts the undisturbed shoreline. Note that not all the panels have the same axes limits.

Looking at the water surface displacements it can be noted that the landslide generates first a positive elevation of the mean sea level, which is even the maximum one close to the generation area (at gauges $12 \mathrm{~S}, 11 \mathrm{~S}$ and $20 \mathrm{~S}$ ). While propagating offshore the first crest decreases and the maximum sea level variation occurs later in the wave train. Moreover this kind of waves presents an irregular shape close where they are generated, and a more regular shape, typical of propagating dispersive waves packet, as far as they radiate offshore.

From the comparison with the laboratory experiments it can be seen that the depth integrated MSE, modified in order to include the wave generation, appears suitable to correctly model the propagation of tsunamis. The water surface elevations and the important properties of waves, as the time of arrival of the crest, the wave heights and periods, are well reproduced. This is especially true if we investigate the far field waves (results at gauges $20 \mathrm{~S}$ to $16 \mathrm{~S}$ ). The numerical model is not able to reproduce exactly the wave field at the gauges close to the generation area, $12 \mathrm{~S}$ and $11 \mathrm{~S}$. Here the water depth is around $10 \mathrm{~mm}$, therefore the same order of magnitude of the wave amplitude. In these condition the linear approximation into the wave equation is no longer valid. From the comparison it can be noted the discrepancy between the measured and the computed free surface elevation, which is mostly due to the wave nonlinearities. Adopting the source term proposed by Tinti et al. (2006) similar consideration can be done and a slight underestimation of the waves amplitude is present at the far field gauges.

The same simulation results are now represented in Fig. 6, in terms of the positive and negative maximum free water surface elevation versus the gauge position distance from the impact point. These maximum and minimum amplitudes refer to the maximum wave encountered (top panel), to the first wave (middle panel) and to the second wave (lower panel). The values of the numerical simulations are normalized with the corresponding measurements in the physical model. Therefore the horizontal lines at values 1 and -1 are the experimental results taken as reference. The circles refer to the solution of Eq. (26), while the black dots to the solution of the same equation with the source term proposed by Tinti et al. (2006). This kind of figure provides a more quantitative evaluation of the models comparisons. It can be noted how the two numerical solutions do not differ from 

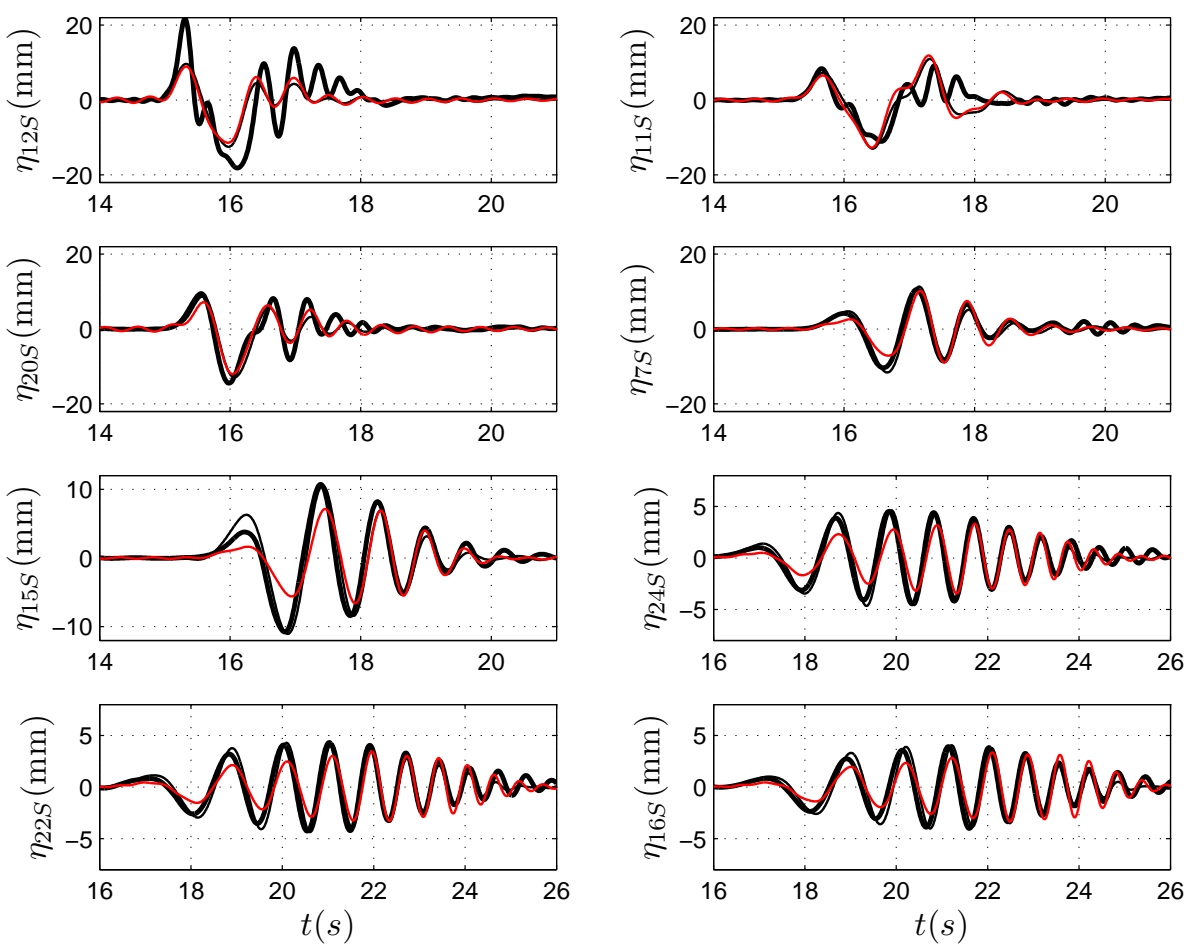

Fig. 5. Free water surface elevation as measured in the laboratory experiments (thick black line), as computed by the numerical model with the proposed source term (thin black line) and as computed with the same numerical model but using the source term proposed by Tinti et al. (2006) (thin red line).
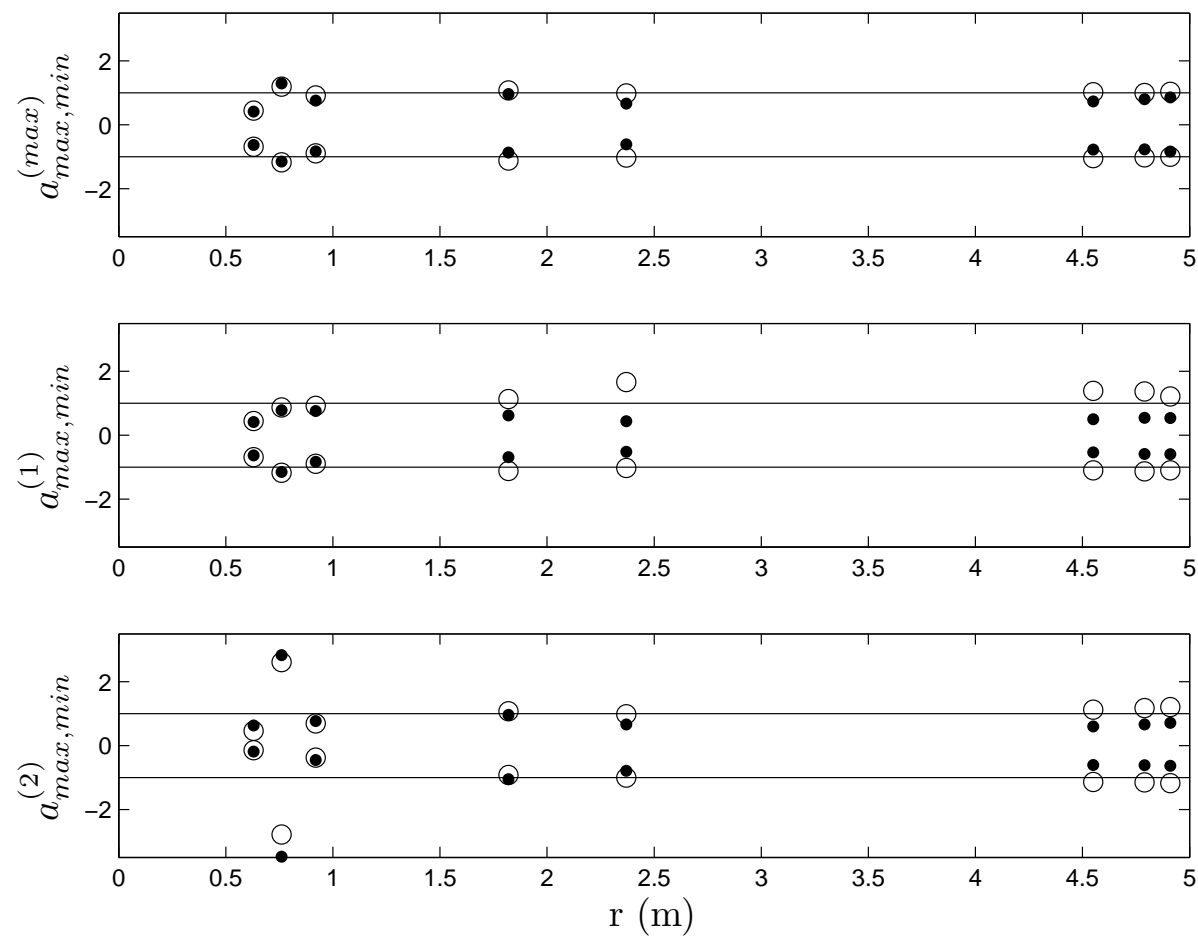

Fig. 6. Positive and negative maximum free water surface elevations values for the maximum (top panel), the first (middle panel) and the second wave (bottom panel) encountered at all the sea level gauges. The circles refer to the values obtained with the proposed model, the dots to the one solved using the source term proposed by Tinti et al. (2006); in both cases the values are normalized with the experimental measurements. 

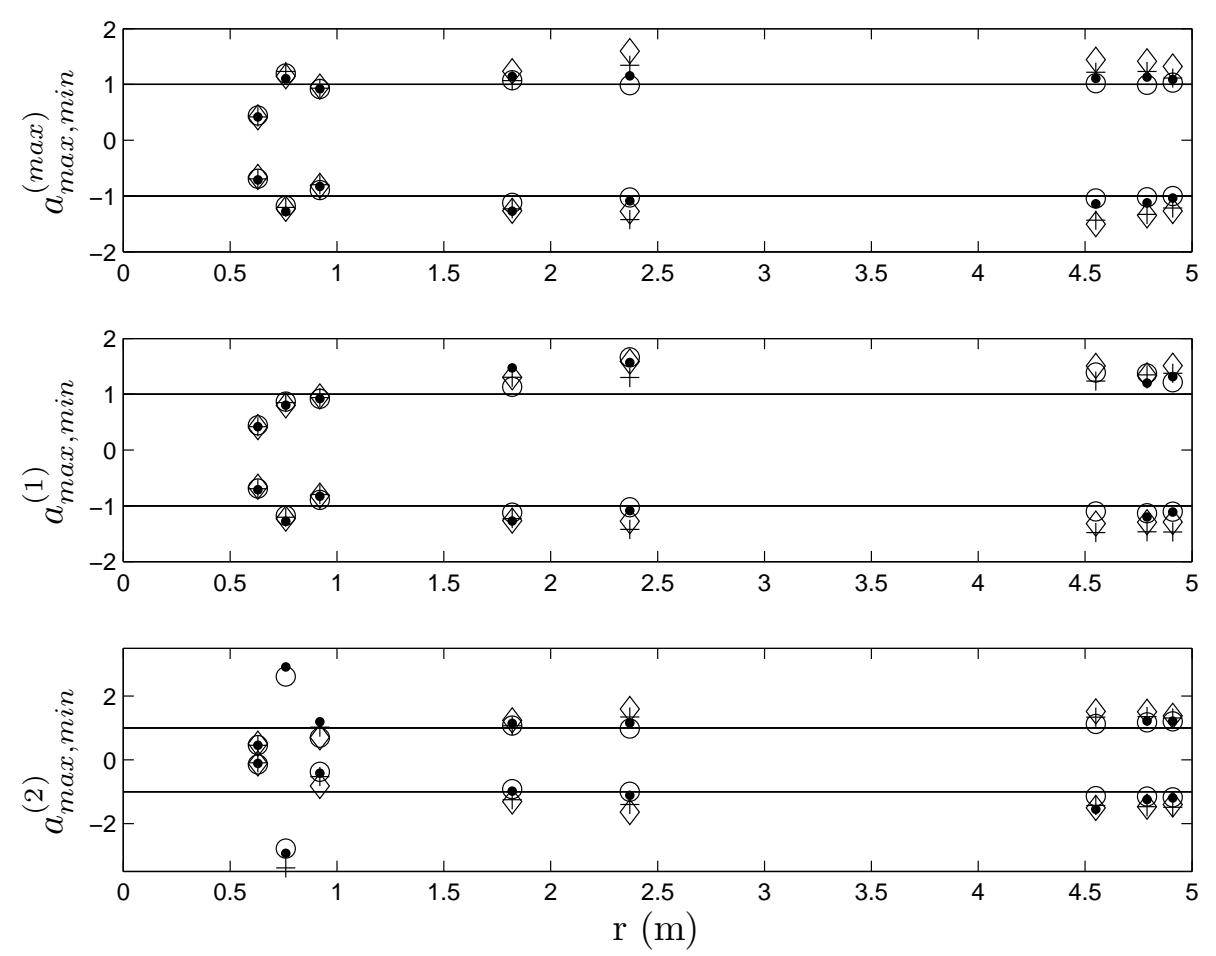

Fig. 7. Positive and negative maximum free water surface elevations obtained from the proposed numerical model for the four landslide kinematics. The different markers refer to the different initial position of the landslide above the undisturbed shoreline: $\zeta=0.30 \mathrm{~m}$ (circle), $\zeta=0.40 \mathrm{~m}$ (point), $\zeta=0.50 \mathrm{~m}$ (plus sign) and $\zeta=0.60 \mathrm{~m}$ (diamond). All these values are normalized with the appropriate experimental measurements.

each other for the first 4 gauges, which are the closest to the generation area. Proceeding further in deeper areas it can be noted small overestimation of the wave amplitude with the proposed source term, while on the contrary the source term proposed by Tinti et al. (2006) appears to slightly underestimate these values. For the present case it can be noted the almost perfect agreement between the numerical and the laboratory results of the maximum free water surface displacement measured in the wave train.

Figure 7 is similar to Fig. 6, but shows the results of numerical computations for different landslide kinematics. As already stated the laboratory experiments were carried out varying the initial landslide position, therefore it changes the landslide velocity because it slides due to gravity acceleration. In this figure the different markers refer to the different initial position of the landslide above the undisturbed shoreline: $\zeta=0.30 \mathrm{~m}$ (circle), $\zeta=0.40 \mathrm{~m}$ (point), $\zeta=0.50 \mathrm{~m}$ (plus sign) and $\zeta=0.60 \mathrm{~m}$ (diamond). All the numerical results shown are normalized with the corresponding experimental results. As in the previous figure, the values for the maximum, the first and the second wave encountered, are reported respectively at the top, middle and bottom panel. From this figure it is possible to evaluate that not a relevant difference exist by varying the landslide kinematics. It can be noted that the numerical model produces a higher overestimation when the landslide starts to move from a higher position (diamond markers). This trend can be explained by remembering that the numerical model reproduces just the waves generated by submarine bottom movements.

In order to further highlight the importance of reproducing the wave frequency dispersion, the same model is applied using the shallow water approximation, i.e. modifying the MSE so that $c=c_{\mathrm{g}}=\sqrt{g h}$. The resulting equation is the forced linear long wave equation, Eq. (25). Since the numerical technique previously described is applied, the field equation is solved in the frequency domain, therefore the Fourier transform of Eq. (25) is used. Figure 8 presents the comparison of the water surface elevations, at the same gauges position, between the physical model (thick line) and the numerical model (thin line). The comparison shows relevant differences in the wave modeling. The forced long wave equation let all the wave frequency components travel at the same velocity, resulting in a single crest-trough wave which propagates with $c_{\mathrm{g}}=\sqrt{g h}$. Moreover the energy content of waves propagating offshore is underestimated, even if the source term in the shallow water equation is the same of the one applied to the MSE. This is due to the fact that shoaling effects reproduced by the long wave equations, when modeling waves from shallow to deep waters, underestimate the wave energy, since the group celerity in deep water is overestimated if assumed equal to $\sqrt{g h}$. 

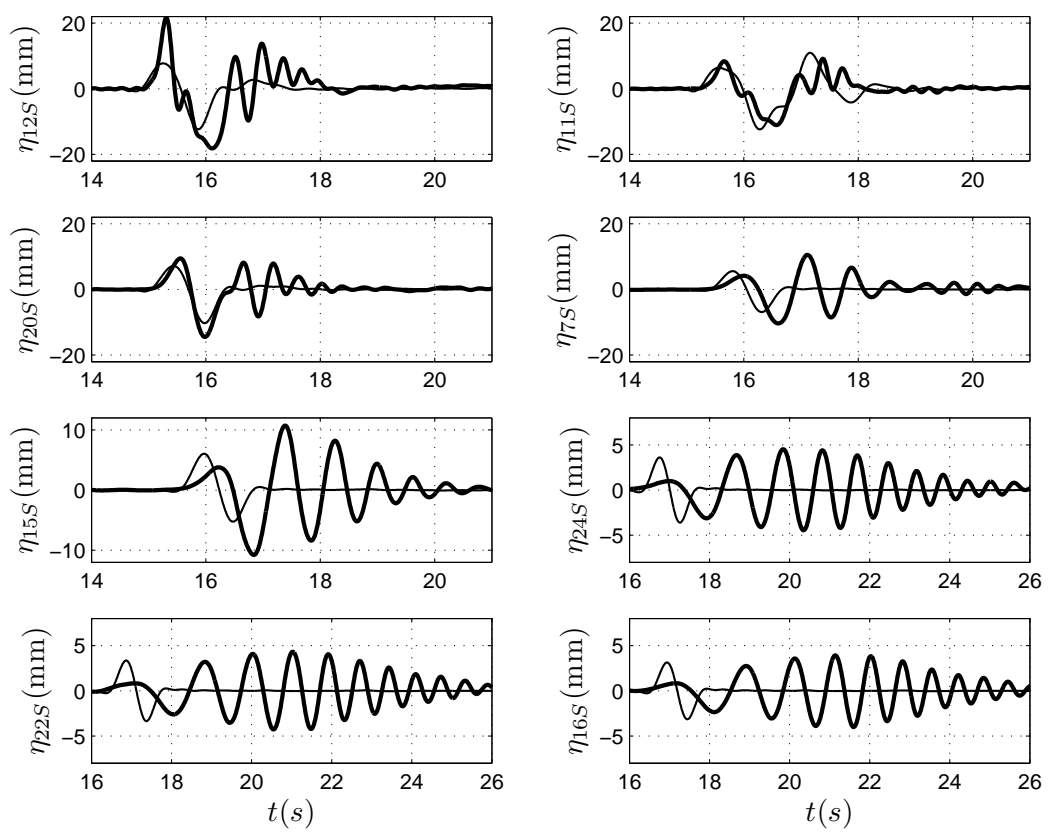

Fig. 8. Free water surface elevation as measured in the laboratory experiments (thick line) and as computed with the same numerical model but in the shallow water approximation (thin line).

Table 2. Values of the maximum wave height and of the first wave height as measured in the physical model and as computed in the presented numerical simulations.

\begin{tabular}{|c|c|c|c|c|c|c|c|c|c|c|}
\hline \multirow[b]{2}{*}{ Gauge } & \multirow[b]{2}{*}{$r(\mathrm{~m})$} & \multicolumn{4}{|c|}{$H^{\max }(\mathrm{mm})$} & \multicolumn{5}{|c|}{$H^{1}(\mathrm{~mm})$} \\
\hline & & Exp. & MSE & MSETinti & SWE & Exp. & MSE & & MSETinti & SWE \\
\hline $12 \mathrm{~S}$ & 0.63 & 39.8 & 22.1 & 20.3 & 20.1 & 39.8 & 22.1 & & 20.1 & 20.1 \\
\hline $11 \mathrm{~S}$ & 0.76 & 19.4 & 20.2 & 19.2 & 18.7 & 19.4 & 20.2 & & 19.2 & 18.7 \\
\hline $20 \mathrm{~S}$ & 0.92 & 23.9 & 21.6 & 19.2 & 17.4 & 23.9 & 21.6 & & 19.2 & 17.4 \\
\hline $7 \mathrm{~S}$ & 1.82 & 19.0 & 19.2 & 19.1 & 12.4 & 14.5 & 16.3 & & 9.7 & 12.4 \\
\hline $15 S$ & 2.37 & 19.1 & 18.9 & 13.7 & 11.3 & 14.5 & 17.3 & & 7.2 & 11.3 \\
\hline $24 S$ & 4.55 & 8.9 & 9.2 & 6.6 & 7.2 & 4.1 & 4.8 & & 2.2 & 7.2 \\
\hline $22 \mathrm{~S}$ & 4.79 & 8.5 & 8.6 & 6.7 & 6.7 & 3.4 & 4.1 & & 2.0 & 6.7 \\
\hline $16 S$ & 4.91 & 7.9 & 7.9 & 6.7 & 6.2 & 3.2 & 3.6 & 1.8 & 6.2 & \\
\hline
\end{tabular}

All these considerations are now collected in a different form, by means of a table. Table 2 presents a quantitative comparison of the wave height of both the maximum and the first wave recorded. In the table are reported the wave height as measured in the laboratory experiments, as obtained solving the present model Eq. (26), as obtained solving again the MSE but with the forcing term as that proposed by Tinti et al. (2006), as computed solving the numerical model in the long waves approximation. Just the values of the maximum wave and the first one encountered are reported because they are considered the most important height parameters of the tsunami wave train. The maximum one because is the more destructive, the first one because, if well estimated, can serve as a warning alert.

\section{Conclusions}

The paper has presented a possible extension of the far field propagation model by Bellotti et al. (2008) to include the tsunami generation phase. As far as the model is based on the linear MSE, a correct reproduction of waves generation and propagation together, occurs just in deep water conditions and for small sea bottom vertical variation. In the present paper is derived the modified MSE, which includes a source term achieved by assuming the time variation of the water depth function, induced by a submarine earthquake or landslide. This source term is function of the generated wave frequency, therefore its inclusion appears natural when solving the wave equation in the frequency domain. However the 
same source term can be applied to numerical models which work in the time domain. In these cases is required a previous filtering of the source term in the frequency domain, and then anti-transformation in the time domain.

The inclusion of the wave source term has been already proposed and validated in the paper by Cecioni and Bellotti (2010). Here for the first time is shown a model validation by comparison with a 3-D laboratory experiment. The physical model reproduces landslide tsunamis generated at the flank of a small conical island, and the experiments have been carried out in order to approximately reproduce a possible tsunami scenario on the volcanic island of Stromboli. The flanks of this island are very steep, therefore when the landslide enters into the sea, it generates waves which are immediately in deep water conditions. The Stromboli tsunami scenario, and therefore the laboratory experiments (Di Risio et al., 2009), appear a study case which satisfy the limits of applicability of the proposed model.

Acknowledgements. This work was carried out under the research projects PRIN 2007 ("Development and validation of hydraulic and geologic tools for supporting a Tsunami Early Warning System. Implementation to the Stromboli Eolie-landslide case.") and FIRB 2008 ("Design, construction and operation of the SMO - Submarine Multidisciplinary Observatory-experiment"), both funded by the Italian Ministry for University and Scientific Research (MIUR).

Edited by: S. Tinti

Reviewed by: two anonymous referees

\section{References}

Bellotti, G., Cecioni, C., and De Girolamo, P.: Simulation of small-amplitude frequency-dispersive transient waves by means of the mild-slope equation, Coast. Eng., 55(6), 447-458, 2008 doi:10.1016/j.coastaleng.2007.12.006

Bellotti, G., Di Risio, M., and De Girolamo, P.: Feasibility of Tsunami Early Warning Systems for small volcanic islands, Nat. Hazards Earth Syst. Sci., 9, 1911-1919, doi:10.5194/nhess-91911-2009, 2009.

Berkhoff, J. C. W.: Computation of combined refraction-diffraction, Proceedings of the 13th International Conference On Coastal Engineering-ASCE, Vancouver, Canada 1972.

Cecioni, C. and Bellotti, G.: Modeling tsunamis generated by submerged landslides using depth integrated equations, Appl. Ocean Res., 32(3), 343-350, doi:10.1016/j.apor.2009.12.002, 2010.

Di Risio, M., De Girolamo, P., Bellotti, G., Panizzo, A., Aristodemo, F., Molfetta, M. G., and Petrillo, A. F.: Landslidegenerated tsunamis runup at the coast of a conical island: new physicalmodel experiments, J. Geophys. Res., 114, C01009, doi:10.1029/2008JC004858, 2009.

Enet, F. and Grilli, S. T.: Experimental Study of Tsunami Generation by Three-Dimensional Rigid Underwater Landslides, J. Waterw. Port C.-ASCE, 133(6), 442-454, doi:10.1061/(ASCE)0733-950X(2007)133:6(442), 2007.
Grilli, S. T., Vogelmann, S., and Watts, P.: Development of a 3D numerical wave tank for modeling tsunami generation by underwater landslides, Eng. Anal. Bound. Elem., 26, 301-313, doi:10.1016/S0955-7997(01)0113-8, 2002.

Grilli, S. T. and Watts, P.: Modeling of waves generated by a moving submerged body. Applications to underwater landslides, Eng. Anal. Bound. Elem., 23, 645-656, doi:10.1016/S09557997(99)0021-1, 1999.

Jiang, L. and LeBlond, P. H.: Three-dimensional modeling of tsunami generation due to a submarine mudslide, J. Phys. Oceanogr., AMS, 24(3), 559-572, 1994.

Liu, P.L.-F., Wu, T.-R., Raichlen, F., Synolakis, C. E., and Borrero, J.: Runup and rundown generated by threedimensional sliding masses, J. Fluid Mech., 536, 107-144, doi:10.1017/S0022112005004799, 2005.

Lynett, P. J. and Liu, P. L. F.: A numerical study of submarinelandslide-generated waves and run-up, P. Roy. Soc. London, 458, 2885-2910, 2002.

Lynett, P. J. and Liu, P. L. F.: A numerical study of the runup generated by three-dimensional landslides, J. Geophys. Res., 110, C03006, doi:10.1029/2004JC002443, 2005.

Okada, Y.: Surface deformation due to tensile faults in a half-space, B. Seismol. Soc. Am., 75(4), 1135-1154, 1985

Peregrine, D. H.: Long waves on a beach, J. Fluid Mech., 27, 815827, doi:10.1017/S0022112067002605, 1967.

Svendsen, Ib A.: Introduction to nearshore hydrodynamics, in: Advanced Series on Ocean Engineering, 24, World Scientific, 2005

Tinti, S., Bortolucci, E., and Chiavettieri, C.: Tsunami Excitation by Submarine Slides in Shallow-water Approximation, Pure Appl. Geophys., 158(4), 759-797, doi:10.1007/PL00001203, 2001.

Tinti, S., Manucci, A., Pagnoni, G., Armigliato, A., and Zaniboni, F.: The 30 December 2002 landslide-induced tsunamis in Stromboli: sequence of the events reconstructed from the eyewitness accounts, Nat. Hazards Earth Syst. Sci., 5, 763-775, doi:10.5194/nhess-5-763-2005, 2005.

Tinti, S., Pagnoni, G., and Zaniboni, F.: The landslides and tsunamis of the 30th of December 2002 in Stromboli analyzed through numerical simulations, B. Volcanol., 68(5), 462-479, doi:10.1007/s00445-005-0022-9, 2006.

Watts, P., Grilli, S. T., Kirby, J. T., Fryer, G. J., and Tappin, D. R.: Landslide tsunami case studies using a Boussinesq model and a fully nonlinear tsunami generation model, Nat. Hazards Earth Syst. Sci., 3, 391-402, doi:10.5194/nhess-3-391-2003, 2003.

Watts, P., Ioualalen, M., Grilli, S. T., Shi, F., and Kirby, J. T.: Numerical simulation of the December 26, 2004 Indian Ocean Tsunami using a Higher-order Boussinesq Model, Ocean Waves Measurement and Analysis, Fifth International Symposium WAVES, Madrid, Spain, 2005.

Wiegel, R. L.: Laboratory studies of gravity waves generated by the movement of a submerged body, EOS T. Am. Geophys. Un., 36(5), 759-74(0), 1955. 